\title{
Numerical tests of dynamical friction in gravitational inhomogeneous systems
}

\author{
A. Del Popolo*
}

\author{
Dipartimento di Matematica, Università Statale di Bergamo, via dei Caniana 2, 24129 Bergamo, Italy \\ Feza Gürsey Institute, PO Box 6 Çengelköy, Istanbul, Turkey \\ Boğaziçi University, Physics Department, 80815 Bebek, Istanbul, Turkey
}

Received 28 January 2003 / Accepted 10 April 2003

\begin{abstract}
In this paper, I test by numerical simulations the results of Del Popolo \& Gambera (1998), dealing with the extension of Chandrasekhar and Von Neumann's analysis of the statistics of the gravitational field to systems in which particles (e.g. stars, galaxies) are inhomogeneously distributed. The paper is an extension of that of Ahmad \& Cohen (1974), in which the authors tested some results of the stochastic theory of dynamical friction developed by Chandrasekhar \& von Neumann (1943) in the case of homogeneous gravitational systems. It is also a continuation of the work developed in Del Popolo (1996a,b), which extended the results of Ahmad \& Cohen (1973), (dealing with the study of the probability distribution of the stochastic force in homogeneous gravitational systems) to inhomogeneous gravitational systems. Similarly to what was done by Ahmad \& Cohen (1974) in the case of homogeneous systems, I test, by means of the evolution of an inhomogeneous system of particles, that the theoretical rate of force fluctuation $\mathrm{d} \boldsymbol{F} / \mathrm{d} t$ describes correctly the experimental one. I find that the stochastic force distribution obtained for the evolved system is in good agreement with the Del Popolo \& Gambera (1998) theory. Moreover, in an inhomogeneous background the friction force is actually enhanced relative to the homogeneous case.
\end{abstract}

Key words. stars: statistics - celestial mechanics - methods: numerical

\section{Introduction}

The study of the statistics of the fluctuating gravitational force in infinite homogeneous systems was pioneered by Chandrasekhar \& Von Neumann in two classical papers (Chandrasekhar \& Von Neumann 1942, 1943, hereafter CN43) and in several other papers by Chandrasekhar (1941, 1943a-e, 1944a,b). The analysis of the fluctuating gravitational field, developed by the authors, was formulated by means of a statistical treatment. In their papers Chandrasekhar \& Von Neumann considered a system in which the stars are distributed according to a uniform probability density, no correlation among the positions of the stars is present and where the number of stars constituting the system tends to infinity while keeping the density constant. Two distributions are fundamental for the description of the fluctuating gravitational field:

1) $W(\boldsymbol{F})$ which gives the probability that a test star is subject to a force $\boldsymbol{F}$ in the range $\boldsymbol{F}, \boldsymbol{F}+\mathrm{d} \boldsymbol{F}$

2) $W(\boldsymbol{F}, \boldsymbol{f})$ which gives the joint probability that the star experiences a force $\boldsymbol{F}$ and a rate of change $\boldsymbol{f}$, where $\boldsymbol{f}=\mathrm{d} \boldsymbol{F} / \mathrm{d} t$.

The first distribution, known as Holtsmark's law (Holtsmark 1919), in the case of a homogeneous distribution of the stars, gives information only on the number of stars experiencing a given force but it does not describe some fundamental features of the fluctuations in the gravitational field such as the speed

* e-mail: adelpop@unibg.it of the fluctuations and the dynamical friction. These features can be described using the second distribution $W(\boldsymbol{F}, \boldsymbol{f})$. Hence, for the definition of the speed of fluctuations and of the dynamical friction one must determine the distribution $W(\boldsymbol{F}, \boldsymbol{f})$. Information on dynamical friction can be obtained from the moments of this last distribution. As stressed by Chandrasekhar $\&$ Von Neumann (1943), for a test star moving with velocity $\boldsymbol{v}$ in a sea of field stars characterized by a random probability distribution of the velocities, $\Phi(\boldsymbol{u})$, we may write:

$\langle\boldsymbol{V}\rangle=\langle\boldsymbol{u}\rangle-\boldsymbol{v}=-\boldsymbol{v}$

where $\boldsymbol{V}$ represents the velocity of a typical field star relative to the one under consideration, $\boldsymbol{u}$ denotes the velocity of a field star. This asymmetry of the distribution of the relative velocities produces, as shown by $\mathrm{CN} 43$, a deceleration of the test star in the direction of motion. This effect is known, from the Chandrasekhar papers, as "dynamical friction". As quoted above, some information on dynamical friction can be obtained by means of the first moment of $\boldsymbol{f}$. As shown by CN43:

$\left\langle\frac{\mathrm{d} \boldsymbol{F}}{\mathrm{d} t}\right\rangle_{\boldsymbol{F}, \boldsymbol{v}}=\frac{-2 \pi}{3} G m n B(\beta)\left[\boldsymbol{v}-\frac{3 \boldsymbol{F} \cdot \boldsymbol{v}}{|\boldsymbol{F}|^{2}} \cdot \boldsymbol{F}\right]$

$\left\langle\frac{\mathrm{d}|\boldsymbol{F}|}{\mathrm{d} t}\right\rangle_{\boldsymbol{F}, \boldsymbol{v}}=\frac{4 \pi}{3} G \operatorname{mn} B(\beta) \frac{\boldsymbol{F} \cdot \boldsymbol{v}}{|\boldsymbol{F}|}$ 
where $m$ is the mass of a field star, $n_{l}$ is the local density, and $B(\beta)$ is definited as:

$B(\beta)=3 \frac{\int_{0}^{\beta} H(\beta) \mathrm{d} \beta}{\beta H(\beta)}-1$

where

$H(\beta)=\frac{2}{\pi \beta} \int_{0}^{\infty} \mathrm{e}^{-\left(\frac{x}{\beta}\right)^{\frac{3}{2}}} x \sin (x) \mathrm{d} x$

and $\beta=|\boldsymbol{F}| / Q_{H}$ where $Q_{H}=\left(\frac{4}{15}\right)^{2 / 3} 2 \pi G M n^{2 / 3}$. These equations show that the amount of acceleration in the direction of $-\boldsymbol{v}$ when $\boldsymbol{v} \cdot \boldsymbol{F} \leq 0$ is greater than that in the direction $+\boldsymbol{v}$, when $\boldsymbol{v} \cdot \boldsymbol{F} \geq 0$ : then the star suffers a deceleration, the a priori probability that $\boldsymbol{v} \cdot \boldsymbol{F} \geq 0$ being equal to the probability that $\boldsymbol{v} \cdot \boldsymbol{F} \leq 0$.

Several authors have stressed the importance of stochastic forces and in particular dynamical friction in determining the observed properties of clusters of galaxies (White 1976; Kashlinsky 1986, 1987) while others studied the role of dynamical friction in the orbital decay of a satellite moving around a galaxy or in the merging scenario (Bontekoe \& van Albada 1987; Seguin \& Dupraz 1996; Dominguez-Tenreiro \& Gomez-Flechoso 1998). Chandrasekhar's theory (and in particular his classical formula see Chandrasekhar 1943b) is widely employed to quantify dynamical friction in a variety of situations, even if the theory developed is based on the hypothesis that the stars are distributed uniformly and it is well known that in stellar systems, the stars are not uniformly distributed, (Elson et al. 1987; Wybo \& Dejonghe 1995; Zwart et al. 1997) in galactic systems as well, the galaxies are not uniformly distributed (Peebles 1980; Bahcall \& Soneira 1982; Sarazin 1988; Liddle \& Lyth 1993; White et al. 1993; Strauss \& Willick 1995). It is evident that an analysis of dynamical friction taking account of the inhomogeneity of astronomical systems can provide a more realistic representation of the evolution of these systems. Moreover from a pure theoretical standpoint we expect that inhomogeneity affects all the aspects of the fluctuating gravitational field (Antonuccio \& Colafrancesco 1994; Del Popolo \& Gambera 1996, 1997; Del Popolo et al. 1996; Gambera 1997). Firstly, the Holtsmark distribution is no longer correct for inhomogeneous systems. For these systems, as shown by Kandrup (1980a,b, 1983), the Holtsmark distribution must be substituted with a generalized form of the Holtsmark distribution characterized by a shift of $W(\boldsymbol{F})$ towards larger forces when inhomogeneity increases. This result was already suggested by the numerical simulations of Ahmad \& Cohen (1973, 1974). Hence when the inhomogeneity increases the probability that a test particle experiences a large force increases, secondly, $W(\boldsymbol{F}, \boldsymbol{f})$ is changed by inhomogeneity. Consequently, the values of the mean life of a state, the first moment of $\boldsymbol{f}$ and the dynamical friction force are changed by inhomogeneity with respect to those of homogeneous systems.

This paper must be intended as the continuation of Del Popolo \& Gambera (1998) paper, in which the study of the effects of inhomogeneity on the distribution functions of the stochastic forces and on dynamical friction was performed.
As anticipated in Del Popolo \& Gambera (1998), the next task to perform was to test the result of the Del Popolo \& Gambera (1998) paper against $N$-body simulations, which is the object of the present paper. The third step (to be developed in a future paper) should be that of finding a formula that describes dynamical friction in homogeneous and inhomogeneous systems only on the basis of statistical theory.

Before continuing we want to stress that when we speak of inhomogeneity we refer to inhomogeneity in position distribution and not to that of velocity distribution. Our work follows the spirit of Kandrup's (1980) in the sense that we are interested in the effect of a non-uniform distribution in the position of stars on the distributions of the stochastic force.

The plan of the paper is the following: in Sect. 2 we review the calculations and formulas needed to obtain $\left\langle\frac{\mathrm{d} \boldsymbol{F}}{\mathrm{d} t}\right\rangle_{\boldsymbol{F}, \boldsymbol{v}}$ in the case of inhomogeneous systems. In Sect. 3, I show how numerical experiments are performed and they are compared with the theoretical results of Del Popolo \& Gambera (1998). Finally, in Sect. 4, I draw my conclusions.

\section{Force derivative and dynamical friction}

The introduction of the notion of dynamical friction is due to $\mathrm{CN} 43$. In the stochastic formalism developed by $\mathrm{CN} 43$ the dynamical friction is discussed in terms of $f$ :

$\overline{\boldsymbol{f}}=\frac{-2 \pi}{3} G m n B(\beta)\left[\boldsymbol{v}-\frac{3 \boldsymbol{F} \cdot \boldsymbol{v}}{|\boldsymbol{F}|^{2}} \cdot \boldsymbol{F}\right]$

where

$B(\beta)=\frac{3 \cdot \int_{0}^{\beta} W(\beta) \mathrm{d} \beta}{\beta \cdot W(\beta)}-1$.

As shown by $\mathrm{CN} 43$, the origin of dynamical friction is due to the asymmetry in the distribution of relative velocities. If a test star moves with velocity $\boldsymbol{v}$ in a spherical distribution of field stars, namely $\phi(\boldsymbol{u})$, then we have that:

$\overline{\boldsymbol{V}}=\overline{\boldsymbol{u}-\boldsymbol{v}}=-\boldsymbol{v}$.

The asymmetry in the distribution of relative velocities is conserved in the final Eq. (6). In fact from Eq. (6) we have:

$\left\langle\frac{\mathrm{d}|\boldsymbol{F}|}{\mathrm{d} t}\right\rangle=\frac{4 \pi}{3} G M n B(\beta) \cdot \frac{\boldsymbol{v} F}{\boldsymbol{F}}$

(CN43). This means that when $\boldsymbol{v} \cdot \boldsymbol{F} \geq 0$ then $\frac{\mathrm{d}|\boldsymbol{F}|}{\mathrm{d} t} \geq 0$, while when $\boldsymbol{v} \cdot \boldsymbol{F} \leq 0$ then $\frac{\mathrm{d}|\boldsymbol{F}|}{\mathrm{d} t} \leq 0$. As a consequence, when $\boldsymbol{F}$ has a positive component in the direction of $\boldsymbol{v},|\boldsymbol{F}|$ increases on average; while if $\boldsymbol{F}$ has a negative component in the direction of $\boldsymbol{v},|\boldsymbol{F}|$ decreases on average. Moreover, the star suffers a greater amount of acceleration in the direction $-\boldsymbol{v}$ when $\boldsymbol{v} \cdot \boldsymbol{F} \leq 0$ than in the direction $+\boldsymbol{v}$ when $\boldsymbol{v} \cdot \boldsymbol{F} \geq 0$.

In other words the test star suffers, statistically, an equal number of accelerating and decelerating impulses. The modulus of deceleration being larger than that of acceleration the star slows down.

Chandrasekhar \& Von Neumann's analysis was extended to inhomogeneous systems in Del Popolo \& Gambera (1998), 
where $W(\boldsymbol{F}, \boldsymbol{f})$ and its first momentum were calculated. Supposing that the distribution function $\tau$ is given by:

$\tau=\frac{a}{r^{p}} \psi\left(j^{2}(M)|\boldsymbol{u}|^{2}\right)$

where $a$ is a constant that can be obtained from the normalization condition for $\tau, j$ a parameter (of dimensions of velocity $\left.^{-1}\right), \psi$ an arbitrary function, $\boldsymbol{u}$ the velocity of a field star. In other words we assumed, according to CN43 and Chandrasekhar \& von Newmann (1942), that the distribution of velocities is spherical, i.e. the distribution function is $\psi(\boldsymbol{u}) \equiv$ $\psi\left(j^{2}(M)|\boldsymbol{u}|^{2}\right)$, but differently from the quoted papers we supposed that the positions are not equally likely for stars, that is, the stars are inhomogeneously distributed in space. A lengthy calculation led us (see Del Popolo \& Gambera 1998 for a derivation and the meaning of symbols) to find that the first moment of $\boldsymbol{f}$ is given by:

$\overline{\boldsymbol{f}}=-\left(\frac{1}{2}\right)^{\frac{3}{3-p}} \cdot A(p) \cdot B(p)^{\frac{p}{3-p}} \cdot \frac{\alpha^{\frac{3}{3-p}} G M L(\beta)}{\pi H(\beta) \beta^{\frac{2-p}{2}}} \cdot\left[\boldsymbol{v}-\frac{3 \boldsymbol{F} \cdot \boldsymbol{v}}{|\boldsymbol{F}|^{2}} \cdot \boldsymbol{F}\right]$

where:

$$
\begin{aligned}
A(p)= & \int_{0}^{\infty}\left[\frac{\sin x}{x^{(4-p) / 2}}-\frac{3 \sin x}{x^{(8-p) / 2}}+\frac{3 \cos x}{x^{(6-p) / 2}}\right] \mathrm{d} x \\
B(p)= & \int_{0}^{\infty} \frac{z-\sin z}{z^{(7-p) / 2}} \mathrm{~d} z \\
L(\beta)= & 6 \int_{0}^{\infty}\left[\mathrm{e}^{(x / \beta)^{\frac{(3-p)}{2}}}\right]\left[\frac{\sin x}{x^{(2-p) / 2}}-\frac{\cos x}{x^{p / 2}}\right] \mathrm{d} x \\
& -2 \int_{0}^{\infty}\left[\mathrm{e}^{(x / \beta)^{\frac{(3-p)}{2}}}\right] \cdot \frac{\sin x}{x^{(p-2) / 2}} \mathrm{~d} x \\
H(\beta)= & \frac{2}{\pi \beta} \int_{0}^{\infty} \mathrm{e}^{-\left(\frac{x}{\beta}\right)^{3 / 2}} \cdot x \sin (x) \mathrm{d} x .
\end{aligned}
$$

The results obtained by us for an inhomogeneous system are different (see Eq. (11)), as expected, from those obtained by $\mathrm{CN} 43$ for a homogeneous system (CN43 - Eq. (105) or Eq. (6)). At the same time it is very interesting to note that for $p=0$ (homogeneous system) our result coincides, as is obvious, with the results obtained by $\mathrm{CN} 43$. In inhomogeneous systems, Eq. (9) can be written, using Eq. (11), as:

$\left\langle\frac{\mathrm{d}|\boldsymbol{F}|}{\mathrm{d} t}\right\rangle=2^{\frac{p}{p-3}} \cdot A(p) \cdot B(p)^{\frac{p}{3-p}} \cdot \frac{\alpha^{\frac{3}{3-p}} G M L(\beta)}{\pi H(\beta) \beta^{\frac{2-p}{2}}} \cdot \frac{\boldsymbol{v} F}{\boldsymbol{F}}$.

In order to check the validity of the quoted relation (Eq. (16)), I have performed numerical experiments. This was done similarly to Del Popolo (1996b) by evolving (now) 100000 points (stars) acting under their mutual gravitational attraction. From the evolved positions and velocities of the stars, $\left\langle\frac{\mathrm{d} \boldsymbol{F}}{\mathrm{d} t}\right\rangle$ was computed as a function of velocity and force, similarly to Ahmad \& Cohen (1974), and then compared with Eq. (16) as I shall describe in the following ${ }^{1}$.

\footnotetext{
${ }^{1}$ In a inhomogeneous system, similarly way to what happens in a homogeneous system, $\boldsymbol{f}$ depends on $\boldsymbol{v}, \boldsymbol{F}$ and $\theta$ (the angle between $\boldsymbol{v}$
}

\section{3. $\mathrm{N}$-body experiments}

To calculate the stochastic force in an inhomogeneous system, I used an initial configuration in which particles were distributed according to a truncated power-law density profile:

$\rho(r)=\rho_{0}\left(\frac{r_{0}}{r}\right)^{p} \quad 0 \leq r \leq R$

(see Kandrup 1980; Del Popolo 1996a). If the velocity distribution is everywhere isotropic then the equation relating the configuration space density $\rho(r)$ to the phase space density $f(E)$ is:

$\rho(r)=4 \pi \int_{U(r)}^{0} \sqrt{2[E-U(r)]} f(E) \mathrm{d} E$

where $U(r)$ is the potential (normalized to zero at infinity). Equation (18) may be converted into an Abel integral equation and inverted, giving the phase space density:

$f(E)=\frac{\sqrt{2}}{4 \pi^{2}} \frac{\mathrm{d}}{\mathrm{d} E} \int_{E}^{0} \frac{\mathrm{d} U}{\sqrt{U-E}} \frac{\mathrm{d} \rho}{\mathrm{d} U}$

(Eddington 1916; Binney \& Tremaine 1987). The initial conditions were generated from the distribution function that can be obtained from Eq. (19) assuming a cut-off radius $R=1$, the mass of the system $M=1, r_{0}=0.15^{2}$ and $G=1$. All the particles had equal mass. To have a system whose total mass is contained in a unitary sphere, Eq. (18) was renormalized and consequently also the potential of the system which is obtained from Eq. (18) through Poisson's equation. The system of 100000 particles was evolved over 150 dynamical times using a tree $N$-body code (Hernquist 1987). During the evolution of the system the essential quantities such as position, force, etc., for each test point of the system was sampled every $\frac{1}{20}$ of a dynamical time (see Del Popolo 1996b; Ahmad \& Cohen 1973 for details). The average of the $\frac{\mathrm{d}|\boldsymbol{F}|}{\mathrm{d} t}$ is a function of velocity, $\boldsymbol{v}$, force, $\boldsymbol{F}$, and the angle between them. The test of Eq. (16) was performed in a similar way to that of Ahmad \& Cohen (1974), namely by integrating out two of the variables and examining $\left\langle\frac{\mathrm{d}|\boldsymbol{F}|}{\mathrm{d} t}\right\rangle$ against the remaining one (see Ahmad \& Cohen 1974). As in Ahmad \& Cohen (1974), $\left\langle\frac{\mathrm{d}|\boldsymbol{F}|}{\mathrm{d} t}\right\rangle_{|\boldsymbol{F}|}$ indicates $\left\langle\frac{\mathrm{d}|\boldsymbol{F}|}{\mathrm{d} t}\right\rangle$ after integrating out the angle and velocity, while $\left\langle\frac{\mathrm{d}|\boldsymbol{F}|}{\mathrm{d} t}\right\rangle_{|\boldsymbol{|}|}$ is $\left\langle\frac{\mathrm{d}|\boldsymbol{F}|}{\mathrm{d} t}\right\rangle$ after integrating out the angle and force. In integrating out the angle, one cannot average over the entire range 0 to $\pi$, since that would give zero. Instead, the cosine was averaged from 0 to $\pi / 2$, and in the numerical experiments only those particles having a cosine in the quoted range were used. In order not to waste the statistics for half the particles the same trick of Ahmad \& Cohen (1974) was used, namely when the cosine is in the range of $\pi / 2$ to $\pi$, the sign of $\frac{\mathrm{d}|\boldsymbol{F}|}{\mathrm{d} t}$ is changed and it is counted in the same statistics. This corresponds to assume that

and $\boldsymbol{F}$ ) while unlike homogeneous systems, $\boldsymbol{f}$ is a function of the inhomogeneity parameter $p$. The dependence of $\boldsymbol{f}$ on $p$ is not only due to the functions $A(p), B(p)$ and to the density parameter $\alpha$ but also to the parameter $\beta=|\boldsymbol{F}| / \boldsymbol{Q}_{H}$. In fact in inhomogeneous systems the normal field $\boldsymbol{Q}_{H}$ is given by $\boldsymbol{Q}_{H}=G M(\alpha B(p) / 2)^{2 /(3-p)}$, clearly dependent on $p$.

2 This is the value I used, remember however that the distribution is scale-free. 


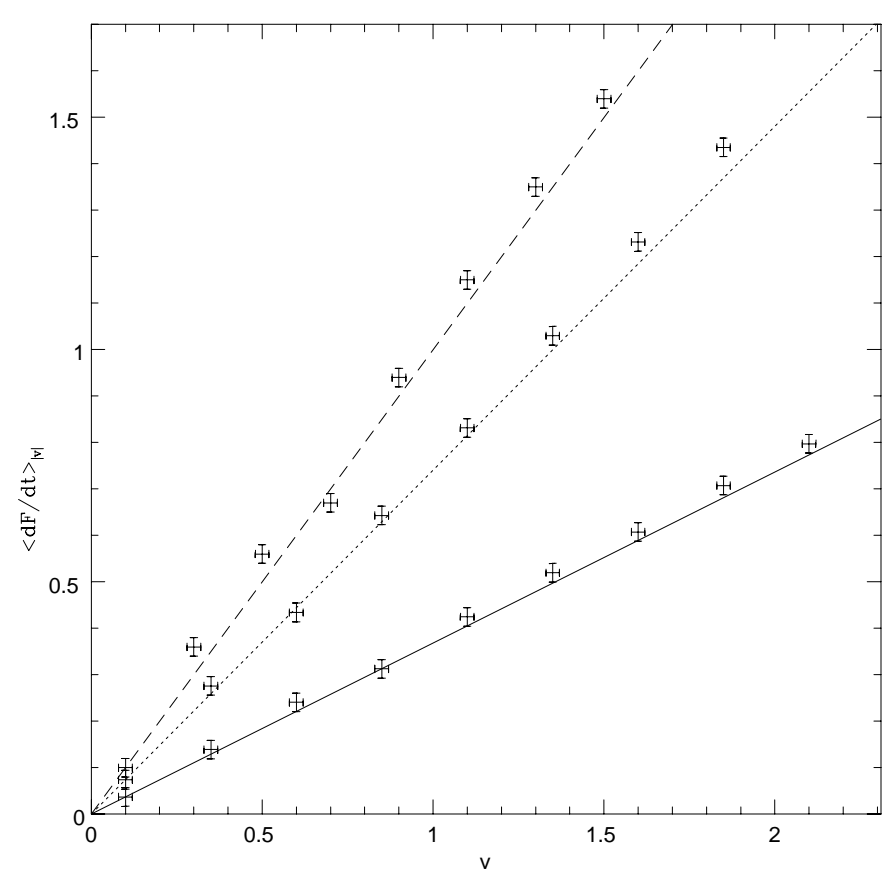

Fig. 1. The average value of the time rate of change of the magnitude of the force as the function of the velocity. The solid line refers to the homogeneous case (Chandrasekhar \& Von Neumann 1943). The dotted and dashed line refers to the cases $p=2.5$ and $p=4$, respectively (see Eq. (26)). Crosses represent the experimental points.

the cosine between $\boldsymbol{v}$ and $\boldsymbol{F}$ is uniformly distributed, which is what is found in numerical experiments. The average value of the cosine between 0 and $\pi / 2$ is $1 / 2$.

For a general distribution $\langle|\boldsymbol{v}|\rangle$ can be calculated as usual:

$\langle|\boldsymbol{v}|\rangle=\int_{0}^{\infty} \frac{f(E) \boldsymbol{v} \mathrm{d} \boldsymbol{v}}{f(E) \mathrm{d} \boldsymbol{v}}$

and $\left\langle\frac{\mathrm{d}|\boldsymbol{F}|}{\mathrm{d} t}\right\rangle$ can be written, in units of $\frac{\left(\frac{1}{2}\right)^{\frac{3}{3-p}}}{\pi} \alpha^{\frac{3}{3-p}} G M\langle|\boldsymbol{v}|\rangle$, as:

$\left\langle\frac{\mathrm{d}|\boldsymbol{F}|}{\mathrm{d} t}\right\rangle_{|\boldsymbol{F}|}=A(p) \cdot B(p)^{\frac{p}{3-p}} \cdot \frac{L(\beta)}{H(\beta) \beta^{\frac{2-p}{2}}}$.

In the particular case of a Maxwellian distribution for velocities:

$\psi=\frac{j^{3}}{\pi^{3 / 2}} \exp \left(-j^{2}|\boldsymbol{v}|^{2}\right)$

where $j^{2}=\frac{3}{2<v^{2}>}$, so that:

$\langle|\boldsymbol{v}|\rangle=\frac{2}{\pi^{1 / 2} j}$

we have that:

$\left\langle\frac{\mathrm{d}|\boldsymbol{F}|}{\mathrm{d} t}\right\rangle_{|\boldsymbol{F}|}=\frac{2^{\frac{p}{p-3}}}{\pi^{3 / 2} j} \cdot A(p) \cdot B(p)^{\frac{p}{3-p}} \cdot \frac{\alpha^{\frac{3}{3-p}} G M L(\beta)}{H(\beta) \beta^{\frac{2-p}{2}}}$

that expressed in units of $\frac{2^{\frac{p}{p-3}}}{\pi^{3 / 2} j} \alpha^{\frac{3}{3-p}} G M$, then Eq. (24) becomes:

$\left\langle\frac{\mathrm{d}|\boldsymbol{F}|}{\mathrm{d} t}\right\rangle_{|\boldsymbol{F}|}=A(p) B(p)^{\frac{p}{3-p}} \cdot \frac{L(\beta)}{H(\beta) \beta^{\frac{2-p}{2}}}$.

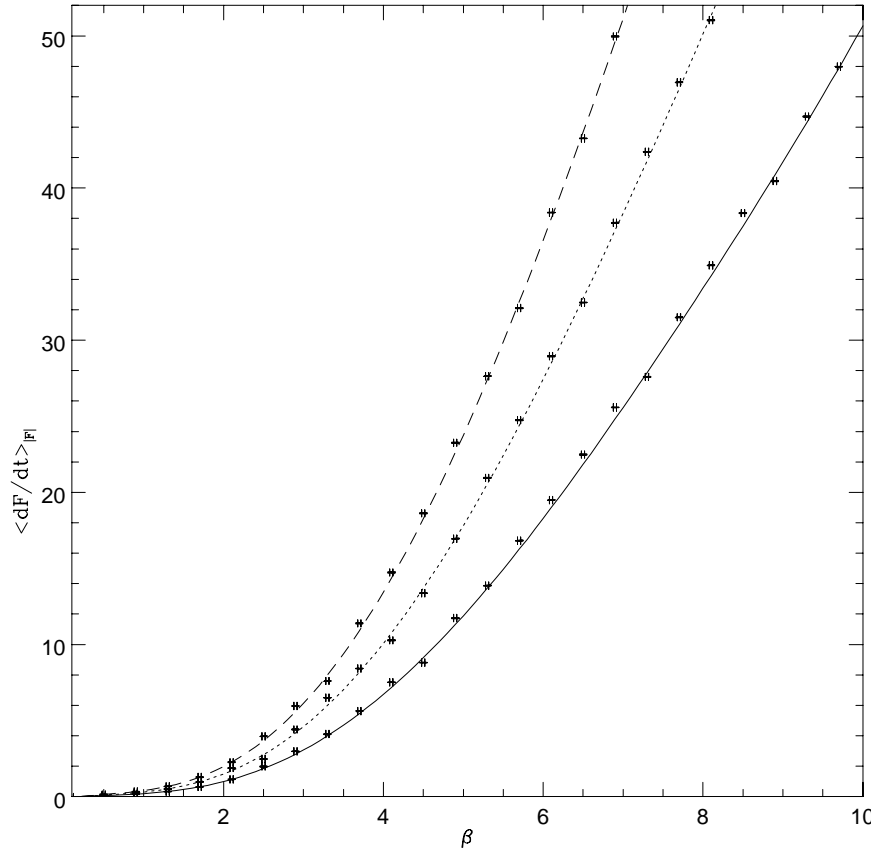

Fig. 2. The average value of the time rate of change of the magnitude of the force as the function of the force. The solid line refers to the homogeneous case (Chandrasekhar \& Von Neumann 1943). The dotted and dashed line refers to the cases $p=2.5$ and $p=4$, respectively (see Eq. (19)). Crosses represent the experimental points.

Similarly to Ahamd \& Cohen (1974), since to integrate out the force from Eq. (16) one has a divergent result, I consider only particles up to a certain maximum value of the force, $\beta_{\max }$ : for example in the case $p=0, \beta_{\max }=10.7$, that involves $97 \%$ of the particles. As observed by Ahmad \& Cohen (1974), any cutoff of the force can be used as long as it is taken into account in both the experiment and the analytic evaluation of $\left\langle\frac{\mathrm{d}|\boldsymbol{F}|}{\mathrm{d} t}\right\rangle_{|v|}$. Defining:

$B_{1}(\beta)=\frac{L(\beta)}{\pi H(\beta) \beta^{\frac{2-p}{2}}}$

and

$\left\langle B_{1}(\beta)\right\rangle=\frac{\int_{0}^{\beta_{\max }} B_{1}(\beta) H(\beta) \mathrm{d} \beta}{\int_{0}^{\beta_{\max }} H(\beta) \mathrm{d} \beta}$

we find, in units of $\left(\frac{1}{2}\right)^{\frac{3}{p-3}}\langle B 1(\beta)\rangle \alpha^{\frac{3}{3-p}} G M$, that:

$\left\langle\frac{\mathrm{d}|\boldsymbol{F}|}{\mathrm{d} t}\right\rangle_{|v|}=A(p) \cdot B(p)^{\frac{p}{3-p}}$.

The results of calculation and numerical experiments are plotted in Figs. 1 and 2. In Fig. 1, I plot the average value of the time rate of change of the magnitude of the force as a function of the velocity. The solid line refers to the homogeneous case while the dotted and dashed lines refer to the cases $p=2.5$ and $p=4$, respectively. Crosses represent the experimental points. As shown, experimental points follow a linear relationship and there is a good agreement with the theoretical prediction (Eq. (21)). In Fig. 2, I plot the average value of the time rate of change of the magnitude of the force as the 
function of the force. As in the previous figure, the solid line refers to the homogeneous case while the dotted and dashed line refers to the cases $p=2.5$ and $p=4$, respectively. In this case, the dependence is no longer linear: it behaves like $B(\beta)$ in the homogeneous case. A comparison with numerical experiments shows that there is a good agreement with the theoretical prediction (Eq. (28)). The situation described in Ahmad \& Cohen (1974), that the experimental data were somewhere in between the theoretical curves for the one-particle and the infinite-particle case, is no longer present and the agreement is better, now. This is due to the larger number of particles used in the simulations. The plots show that Chandrasekhar \& Von Neumann's theory of dynamical friction in gravitational systems gives a good description of experimental data (solid line and data), and so does the generalization of the quoted theory to inhomogeneous systems (dotted line, dashed line, and respective data). In inhomogeneous systems, Chandrasekhar's result which relates the frictional force only to the local properties of the background at the position of the object, is no longer true, and friction depends on the global structure of the system. This point is in agreement with Maoz (1993), who showed that in inhomogeneous media the friction, unlike Chandrasekhar's formula, depends on the global structure of the entire mass density field.

\section{Conclusions}

In this paper, I tested by numerical simulations the results of the Del Popolo \& Gambera (1998) paper, dealing with the average value of the time rate of change of the magnitude of the stochastic force in inhomogeneous gravitational systems. In agreement with Ahmad \& Cohen (1974), the stochastic theory of dynamical friction developed by Chandrasekhar \& Von Neumann (1943), in the case of homogeneous gravitational systems, gives a good description of the results of numerical experiments. The stochastic force distribution obtained for inhomogeneous systems, obtained by Del Popolo \& Gambera (1998), is also in good agreement with the results of numerical experiments. Finally, in an inhomogeneous background the friction force is actually enhanced relative to the homogeneous case.

Acknowledgements. I am grateful to E. N. Ercan and G. Mamon for stimulating discussions during the period in which this work was performed. I would like to thank Boğaziçi University Research Foundation for the financial support through the project code 01B304.

\section{References}

Ahmad, A., \& Cohen, L. 1973, ApJ, 179, 885

Ahmad, A., \& Cohen, L. 1974, ApJ, 188, 469

Antonuccio-Delogu, V., \& Colafrancesco, S. 1994, ApJ, 427, 72

Bahcall, N. A., \& Soneira, R. M. 1982, ApJ, 262, 419

Bontekoe, T. R., \& van Albada, T. S. 1987, MNRAS, 224, 349

Binney, J., \& Tremaine, S. 1987, Galactic Dynamics, in Princeton Series in Astrophysics (Princeton University Press)

Chandrasekar, S. 1941, ApJ, 94, 511

Chandrasekar, S. 1943a, Rev. Mod. Phys., 15, 1

Chandrasekar, S. 1943b, ApJ, 97, 255

Chandrasekar, S. 1943c, ApJ, 97, 263

Chandrasekar, S. 1943d, ApJ, 98, 25

Chandrasekar, S. 1943e, ApJ, 98, 47

Chandrasekhar, S. 1944a, ApJ, 99, 47

Chandrasekhar, S. 1994b, ApJ, 99, 25

Chandrasekhar, S., \& Von Neumann, J. 1942, ApJ, 95, 489

Chandrasekhar, S., \& Von Neumann, J. 1943, ApJ, 97, 1 (CN43)

Del Popolo, A. 1996a, A\&A, 305, 999

Del Popolo, A. 1996b, A\&A, 311, 715

Del Popolo, A., \& Gambera, M. 1996, A\&A, 308, 373

Del Popolo, A., \& Gambera, M. 1997, A\&A, 321, 691

Del Popolo, A., Gambera, M., \& Antonuccio-Delogu, V. 1996, Mem. Soc. Astr. It., 67, 957

Del Popolo, A., \& Gambera, M. 1998, A\&A, 342, 34

Dominguez-Tenreiro, R., \& Gomez-Flechoso, M. A. 1998, MNRAS, 294, 465

Eddington, A. S. 1916, MNRAS, 76, 572

Elson, R., Hut, P., \& Inagaki, S. 1987, ARA\&A, 25, 565

Hernquist, L. 1987, ApJSS, 64, 715

Holtsmark, P. J. 1919, Phys. Z., 20, 162

Kandrup, H. E. 1980a, Phys. Rep., 63, 1

Kandrup, H. E. 1980b, ApJ, 244, 1039

Kandrup, H. E. 1983, Ap\&SS, 97, 435

Kashlinsky, A. 1986, ApJ, 306, 374

Kashlinsky, A. 1987, ApJ, 312, 497

Liddle, A. R., \& Lyth, D. H. 1993, Phys. Rep., 231, 2

Maoz, E. 1993, MNRAS, 263, 75

Peebles, P. J. E. 1980, The large scale structure of the Universe (Princeton: Priceton University Press)

Sarazin, C. 1988, X-ray emission from Clusters of Galaxies (Cambridge: Cambridge Univ. Press)

Seguin, P., \& Dupraz, C. 1996, A\&A, 310, 757

Strauss, M. A., \& Willick, J. A. 1995, Phys. Rep., 261, 271

White, S. D. M. 1976, MNRAS, 174, 19

White, S. D. M., Briel, U. G., \& Henry, J. P. 1993, MNRAS, 261, L8

Wybo, M., \& Dejonghe, H. 1995, A\&A, 295, 347

Zwart, S. F. P., Tout, C. A., \& Lee, H. M. 1997, in Highlights of Astronomy, ed. J. Andersen (Kluwer Academic Publishers), 11 